in many, but not in all cases, causing endarteritis, leucocytic exudation, neoplastic organization and capillary extravasation into the cerebral tissues comparable to the rosacea observable in some drunkards' cheeks and noses. In my autopsies of the alcoholic insane at the county asylum, I invariably noticed a. rusty discoloration of the dura mater along the course of the superior longitudinal sinus, and other evidences of old inflammatory conditions such as adhesions of the membranes and cerebral tissue of the convexity and basilar regions. The vascular and meningeal alterations varied in degree according to the patient's age. Where frailty of blood veseel organization existed congenitally there was greater liability to pathologic change in such cases.

The finer mental coördinations in any one are main tained by effort; being the latest faculties acquired, and their tenure being so dependent upon full brain integrity, it is plain that the moral nature has been superimposed upon the less easily destroyed brute nature, through finer and weaker histologic arrangements acquired and inherited, demanding for their exercise the clearest kind of brain activity. Vitiated blood quickly blots out these better but feebler functions for the time being, just as exhaustion is felt first in our weakest joints. So the moral nature, which is merely a higher intelligence, may depart when the seat of intellect is weakened by any cause such as senility, drinking, insanity, arrest of development, traumatism and some diseases.

When certain pathologic adjustments, involving imperfect compensations, occur, such as thickened arterial walls which resist the increased flow of blood, then a new plane of mental operation is established, which, if disturbed by change of habits, as by withdrawal of the customary greater heart impulse, it is but partially and inadequately recompensated by the pure blood. Practically, the adjacent cerebral tissue must suffer from anemia to a greater or lesser extent, and where previously the blood was driven through disarranged avenues, it now makes its way feebly and in places not at all. Nor is this all; the sclerosed and otherwise changed tissue becomes a more prominent hindrance to function when the artificial nutrition and circulation is cut off. So the poor fool of a drunkard is too often thus "damned if he does, and damned if he don't'. continue.

Summarizing post-alcoholic bodily and mental states there may be found many organic destructive changes in the blood vessels, liver, nerves and brain, which were not so evident during the addiction, owing to the somewhat compensatory effect of the alcohol, and hence the masking of diseases.

The simple privation may kill through the weak heart losing its wonted stimulant, but such cases are not very common; debility is the most frequent consequence of "reform," but this is often a return to what preceded and may have led to the over-indulgence. Cerebral blood vessels subjected to engorgement are liable to rupture at any time where weak points exist, and fatty degeneration of vessel walls, induced by the liquor, may culminate in apoplexy, whether drinking is continued or not.

A single severe attack of delirium tremens may make profound changes for the worse in the future workings of the brain, and the typhoid stage of some cases of delirium tremens show the ravages of the poison often in life-long sequelæ.

Chronic alcoholic insanity may make its first gross appearance after abstinence enforced in jail or otherwise.

Necessarily, when liquor is withdrawn, a change for the better is ordinarily the rule, but such withdrawal in some cases may operate as a shock, and in all cases a readjustment of the entire physiologic make-up must occur. It is conceivable that epilepsy or insanity may find in such shock a potent exciting cause and the whisky soaking is ample as a predisposing influence, when it can alter the brain structure, in time, as thoroughly as a contusion or a concussion.

But what the inebriate has drank, how long he has been drinking, and his power of resistance, associated diseases, hereditary and other tendencies, are to be taken into account; and with these it is surprising how large a number of heavy drinkers escape any obvious trouble due to such excesses. A well-known druggist of Chicago was a sot until his fortieth year, stopped drinking and died at 70 years, having built up a large business; while others who had not taken half his risks with liquor succumbed during or after ceasing their bad habits.

Post-alcoholic conditions such as insanity, paralyses, weak heart, etc., that occur in a minority of cases after alcoholic disuse, only the most thoughtless or perverted could use as arguments against the stopping of drink; as the liquor in such cases produced the trouble which merely culminated after the habits were changed; such climax, being inevitable in any case, and impending, might have been reached earlier, or in a graver form, had the inebriety continued.

In a few words, drunkenness is a constant menace to the mental and bodily health, and it is far safer to escape from its ravages, scarred and maimed, than to go on sooner or later to certain destruction. Though the vast majority may be rescued entire, or nearly so from intemperance, no one can tell what the chemic devil has left of him until months or years of sobriety have passed.

\section{THE ESSENTIAL OF INSANITY.}

BY J. SANDERSON CHRISTISON, M.D.

FORMERLY OF THE NEW YORK CITY ASYLUMS FOR THE INSANE, BLACKWELL'S ISLAND AND WARD'S ISIAND.

CHICAGO.

If by insanity we simply mean more or less deviation from the free normal action of the mind, without regard to cause, then, indeed, no mortal is constantly free from it. For as mental activity, in degree and range, is a measure of physical tone and proportion, especially of the brain, it is quite evident the accidents of existence preclude a constant normal. But the term insanity, to the popular mind implies the state of personal irresponsibility and the question in regard to a particular act, is the proof of the existence of this state. The law, with ref $\epsilon$ rence to a specific act, simply demands, Was he sane or was he not? implying by the question either a fracture of mental integrity or a degree of derangement beyond which responsibility justly ceases.

It is quite clear that from a practical standpoint, we can not hold to the position that all men are insane or that all abnormal states of the mind are degrees of insanity, though leading to it. Just as we do not recognize tuberculosis until the bacillus is in the field, so we may have an essential characteristic of insanity. That essential of insanity is a delusion 
uninfluenced by evidence sufficient to refute and due to disease of the brain. This definition includes a much wider range than asylum wards and embraces a class to whom we might hesitate to impute the charge, while it drops others into the still wider circle of cranks and crippled creatures. But to maintain that a man is not really a lunatic unless he holds a delu. sion will depend upon what we regard a delusion to be. An insane delusion is a false concept-an idea which is the product of defective data and defective reasoning and held in spite of the presentation of a corrected data and corrected reasoning. This distinguishes it from a common delusion-an error of belief remediable by suitably put evidence.

A common or simple delusion is due more to educational errors, but which by necessity-from the aberrant nature of errors-they more or less induce material conditions off the normal line, and thus, in addition to the direct evil of their falsity, they indirectly conduce to undermine the physical tore requisite for perfectly free mental action. But the lesser degree of defect may be in the course of the evolution, of the greater, yet so common is it that few of us could plead freedom from a finding of some sort.

Insanity has no direct relationship to the size or shape of the brain, but only to its lack of tone or integrity-a molecular disintegration-which in its last analysis is the registration of conduct and which ought to be in line with a motive consistent with common sense-the normal product of common experience. A simple error of belief, a mere notion or faint suspicion, may so mislead as to react injuriously before correction is made, or they may even become the substructure of a slowly constructed system of beliefs having a degree of plausibility which may earn for its advocate a respectful attention. As one error held begets another, and as all errors, when actuating, mislead in conduct which determines the tone of the organs of mind, it is evident the result of the one becomes a cause of the other, and thus we may have insidiously developed a fixed delusion or systematized set as in a Swedenborg or a Schweinfurth. If the tone of the brain is fully at par, the action of the mind will be accurate as far as attention is directed both as regards the nature, number and order of perceptions or sensations and the process of reasoning on their relationship and thus correct conception result. It will be the truth, if not the whole truth. A greater brain and one more perfectly proportioned would supply the energy, other things being equal, for more extended attention, and thereby a more comprehensive result.

Systematic observations in prison and asylum practice in New York city abundantly satisfied me that neither mere shape nor size of brain had anything to do with insanity or criminality. But it was evident that the causative conditions of shape and size did have to do with capacities or talents which, did they not meet a fitting environment, would at least negatively contribute to loss of brain tone from the simple lack of proportional exercise, and in this way lead to demoralization, which, of course, always induces disease or a receptivity for it in some degree.

The delusions of illusion and hallucination differ from the purely relational-those wholly due to ideational incompetence-only in being less centric in origin. They are more fleeting and susceptible of correction, but they may be just as strongly actuating while present. Every delusion is an immediate product of defective ideation-the intellectual act involving perception, reason and will and expressing a failure of the brain. When illusion and hallucination are absent, the delusion is purely the product of ideational incoherence-an incompetent relationship of the ideas involved. The faculties are subjective and the emotions are irregular and either excessive or greatly reduced, with egotism, suspicion, jealousy or fear made manifest. These states are always symptomatic of cerebral instability and although so common, they can not exist in the perfect individual who necessarily has a clear conscience and correct appreciation of facts within the range of his vision, which is serene at all times. Their combination and form of associated delusion are formed by the elements in the history of the individual. Great depression or great excitement can not exist without delusion, for the mental condition is such that ideas can not be correctly associated and thus, if the stage of confusion is transcended, some insane delusions arise, although they may only be fleeting. If correct facts in correct form or relationship were present in consciousness, such morbid manifestations could not exist any more than fire can without fuel. Egotism, jealousy, suspicion, hatred, envy, anger and fear are all expressions of morbid condition or weakness, though we regard them in moderation as perfectly natural. They are due to imperfect comprehension of facts before the mind and which is a measure of lost energy. In delusion, the faculty of attention is so weakened that the presentation of the truth is not appreciated.

The emotions are the product of subconscious ideational activity, made conscious in the form of feeling instead of image, and their precise disposition will accord with the data of the memory-the purely conservative faculty-but only those data which are in affinity with the exciting agent. Emotion is inversely proportionate to intellectual range and is the expression of the inability of the intellect to take distinct and immediate cognizance of all the ideas in the memory aroused by the excitant, just as it fails to distinctly perceive all sensory impressions from the outside. This is a normal condition, but is very often out of proportion long before derangement to the degree of insanity is reached.

By the free normal action of the mind, I do not mean an everyday type of health, but a much higher. To be on a level with a certain class or race of humanity, may be a long way below the highest possibilities of individual capacity. Indicative of this fact is the common lack of quickness, preciseness and completeness of mental action within the range of individual knowledge and capacity. Our criterion should be formed from ultimate motives for existence. Insanity is not a relative term, but a positive. It is an extreme aberration from the normal, and the normal is only normal in so far as it is in correct line with evolution or final purpose in nature and not simply in harmony with immediate social environment.

An insane delusion has three components, error of fact, incapacity for correction, error of inference and illogical tenacity. The responsible faculty for this is the attention which is so affected by a weakened will that it can not be held long enough to complete perception and conception, and ideational 
displacement results. But as the will is the dominant $\mid$ many seem to think that with a given specific remedy power of the mind and owes its strength to support for any given disease, all that is necessary is to apply from the other faculties, it is evident that its weak- that remedy mechanically, without respect to anyness is due to a general loss of energy, which sub- thing else, physiologic or pathologic. Chronic tuserves the mind as a whole, and as a consequence thus becomes an added cause for farther evil.

The evidence of a delusion is not always readily recognized, even by asylum physicians. Some subjects secrete their delusions; some have their delusions instantly dissipated by the presence of a physician, officer or stranger; some only have momentary delusions or perhaps frequently changed; some change with prominent events; some have delusions so buried that they can only be elicited by particular conditions and requiring the analytical skill of an expert.

But the point of present issue is that where there is no delusion, there is no insunity, for the mind that is competent to comprehend facts and their bearings within the scope of its education and the limits of ordinary surroundings is a mind capable of correction on any error; and, conversely, a mind that is not thus competent must of necessity beget delusion of one form or another which it is as incompetent to discharge, no matter what the evidence combating.

\section{FURTHER REPORT OF THE SERLMTHERAPY IN TUBERCULOSIS.}

BY PAUL PAQUIN, M.D.

IHYSICIAN-IN-CHIEF ST. LOUIS SANITARILM FOR THROAT AND LLNG DISEASES.

$$
\text { sT. LouIs, yo. }
$$

Up to this date all of the reports which I have had the honor to make to the medical profession, bearing on the use of the horse-blood serum in the treatment of tuberculosis, cover a number of cases in which the improvement had been more or less marked. Our experiments up to the last report had not gone far enough to justify the statement that we had, in fact, cases of tuberculosis which had been cured. The present writing is chiefly for the purpose of reporting results of a more positive nature and, at the same time, to say a few words on the complications that may arise during serum injections; the conditions which militate against its use; also the extent, so far as I am able to present from past experience, of the expectations that may be based on the new treatment. I will begin with the latter propositions.

In the first place, one should always bear in mind that tuberculosis varies a great deal with individuals and with respect to the organs affected. Pulmonary tuberculosis itself presents a wide range of pathologic conditions that must be judged and interpreted according to the extent and nature of each, locally and generally on the system. Consequently, no two cases of pulmonary consumption can, with safety, and hope of success, be treated alike by any one remedy, irrespective of the general and local constitutional or organ disturbances. One would suppose that a specific treatment should apply to all forms of it. One would think that if creasote is good in one case it ought to be in another. If inhalation of essential oils does good in one case it should benefit in another. If serum injections produce good results in a given case, they should have similar effects, irrespective of any other treatment, in any case, affecting any organ.

But such is not the case. It is unfortunate that so tubercles th of them), each surrounded by a special envelope, impenetrable by the circulation or by medicines. These tubercles may be surrounded by acute inflammatory processes or free from them. Some may be in an advanced stage and others just forming. In such cases, the opening of the tubercles happens gradually and successively; that is, some of them break to-day and others to-morrow or later on. Consequently, a more or less constant process of eruption goes on for months or years. Sometimes eventually, cavities are formed, during which certain principles of the morbid matter are partly absorbed, but mostly expelled by expectoration. It is evident that no form of treatment can arrest these slow cases promptly. The good results of serumtherapy in these have been slow in proportion to the nature of the affection, but have occurred, and we have under observation patients of this class who are unquestionably steadily improving, if we are to judge by the disappearance of the chief symptoms, such as night sweats, fever, loss of appetite, emaciation. Indeed, to arrest the progress of the disease in such cases is itself progress toward a cure. In another form of tuberculosis, one may meet with contracted lungs, sometimes with alterations of a more or less fibrous character, lungs encased usually in a narrow or otherwise defective chest. These cases are also very slow (they do not always end with cavities), and serumtherapy as well as other agents must naturally fail to bring quick improvement.

It is only slowly that one can reach such patholugic lesions, whatever agent is used.

On the other hand, we find chronic cases of tuberculous processes existing here and there, or localized in one apex or both apices, which are of a subacute character, and around which there exists more or less infiltration which, under physical examination, simulate to some degree the fibrous consolidations which I have mentioned before. This form of tuberculosis yields more readily to the serumtherapy than those above mentioned.

Again, there are cases of chronic tuberculosis of the lungs in which the tuberculous alterations con. sist first in localized inflammatory processes, unknown sometimes to the individual until a hemorrhage takes place and which sometimes end in cavities more or less extensive, and more or less serious according to their location, etc. One occasionally meets with such cases without detecting any other alterations in the lungs.

It must be apparent to any physician that the various conditions just mentioned (which do not by any means cover all the types of pulmonary phthisis) are not to be treated by any stereotyped formula and serotherapy, acting as a specific agent, should not be depended upon exclusively, irrespective of other conditions in the diseased organization. In other words, every case should be managed scientifically, using the serum only for the purpose it is intended, namely, to give to the organism the physiologic elements which it lacks to fight the tuberculous process from within.

Most of the chronic forms are mixed infections, 